'Laboratorio de Biología

Molecular y Celular del Cáncer, Departamento de Ciencias Biomédicas, Facultad de

Medicina, Universidad Católica del Norte. Coquimbo, Chile. ${ }^{2}$ Departamento de Ciencias Biomédicas, Facultad de

Medicina, Universidad Católica del Norte. Coquimbo, Chile. 3Unidad de Anatomía Patológica, Hospital San Pablo de Coquimbo. Coquimbo, Chile.

${ }^{4}$ Departamento de Clínica, Facultad de Medicina, Universidad Católica del Norte. Coquimbo, Chile.

${ }^{5}$ Departamento de Salud Pública, Facultad de Medicina, Universidad Católica del Norte. Coquimbo, Chile. aioquímico.

bEstudiante Kinesiología. 'Enfermera, Dr. Enfermería.

dBioquímico, PhD en Ciencias Biomédicas.

Trabajo Financiado por CORFO 12IDL2-16202. Autores declaran no tener conflictos de interés.

Recibido el 11 de diciembre de 2017, aceptado el 31 marzo de 2018.

Correspondencia a: Dr. Giuliano Bernal Laboratorio de Biología Molecular y Celular del Cáncer (CáncerLab) Departamento de Ciencias Biomédicas, Facultad de Medicina. Universidad Católica del Norte. Larrondo 1281, Coquimbo, Chile. Teléfono: 56-51-2205988 gbernal@ucn.cl

\section{Prevalencia de cepas cagA-positivo en la región de Coquimbo, determinada mediante nested-qPCR en muestras fecales}

\author{
TRACY WORMWOOD ${ }^{1, a}$, ÁLVARO PARRA ${ }^{1, b}$, \\ GUSTAVO BRESKY ${ }^{2}$, JUAN A. MADARIAGA ${ }^{2,3}$, SERGIO HÄBERLE ${ }^{4}$, \\ JACQUELINE FLORES ${ }^{5, \mathrm{c}}$, GIULIANO BERNAL ${ }^{1,2, \mathrm{~d}}$
}

\section{Frequency of CagA-positive Helicobacter pylori strains in 160 patients subjected to endoscopy}

Background: Helicobacter pylori is the most significant pathogen associated with gastric diseases, including gastric cancer. Infected patients with strains that are CagA-positive generally have worse outcomes than those infected with $\mathrm{Ca}$ gA-negative strains. Patients infected with CagA-positive strains have a higher risk for developing gastric cancer. Aim: To determine the prevalence of CagA-positive H. pylori strains in fecal samples of patients from the Coquimbo Region of Chile, using a non-invasive, nested-qPCR method. Material and Methods: We evaluated 160 patients with gastrointestinal symptoms subjected to an upper gastrointestinal endoscopy. DNA was extracted from fecal samples and tested for the presence of $H$. pylori using nested-qPCR for the ure C gene, and subsequently compared with the results of histology-Giemsa stain from the patients' endoscopic biopsies. When H. pylori was found, the presence of CagA-positive strains was determined via nested-qPCR. Results: The histology-Giemsa stain was positive for $H$. pylori infection in 123 patients (76.9\%), while the analysis of fecal samples detected $H$. pylori in 129 patients (80.6\%). The sensitivity and specificity of nested-qPCR to detect the bacterium was 96.7 and $73.0 \%$ respectively. Among patients with the infection, 25\% had CagA-positive strains. Conclusions: In this sample of patients, there is a low prevalence of CagA-positive H. pylori strains.

(Rev Med Chile 2018; 146: 596-602)

Key words: Bacterial infections; Feces; Helicobacter pylori; Molecular Epidemiology.
L a infección por Helicobacter pylori, afecta casi a $50 \%$ de la población mundial y puede llegar a $90 \%$ en países subdesarrollados, siendo una de las infecciones crónicas más importantes en la especie humana. Incluso, dentro de un mismo país, la prevalencia puede variar significativamente entre las poblaciones urbanas de mayor nivel económico y las poblaciones rurales ${ }^{1}$. En Chile, la prevalencia de infección por esta bacteria, supera $75 \%$ entre la población adulta asintomática ${ }^{2}$, siendo una de las tasas más altas en el mundo ${ }^{3,4}$.

La trasmisión de H. pylori ocurre principalmente de persona a persona por vía fecal-oral, oro-oral, o gastro-oral, y no se ha identificado un reservorio ambiental significativo ${ }^{5,6}$.

La infección por $H$. pylori se asocia a gastritis superficial, la cual progresaría hacia gastritis atrófica crónica, metaplasia intestinal, displasia, y finalmente cáncer gástrico ${ }^{7-9}$. Con estos antecedentes, 
H. pylori ha sido clasificado como un carcinógeno tipo I, desde 1994, de acuerdo a la Organización Mundial de la Salud ${ }^{10}$. Al respecto, la virulencia de $H$. pylori se asocia fuertemente a la presencia de la isla de patogenicidad cag (cagPAI) que codifica a la oncoproteína CagA ${ }^{11}$. Esta proteína es translocada al citoplasma de la célula gástrica a través del sistema de secreción tipo IV (T4SS), codificado en el mismo cag-PAI ${ }^{12}$, en donde puede conducir la activación de cascadas de señal involucradas en el aumento de la proliferación celular, apoptosis y supresión del ciclo celular ${ }^{13}$. La región C-terminal de CagA contiene una secuencia de cinco aminoácidos, conocida como motivo EPIYA (GluPro-Ile-Tyr-Ala), y de los cuales se han descrito cuatro de ellos, denominados EPIYA-A, -B, -C, y $-D$, los que han servido para clasificar a las cepas cagA-positivo de $H$. pylori, y los responsables del mayor potencial carcinogénico de estas cepas ${ }^{14}$.

Recientemente, nuestro laboratorio ha desarrollado un método diagnóstico, basado en nested-qPCR, para determinar la presencia de $H$. pylori en muestras fecales humanas ${ }^{15}$. Este método diagnóstico, que mide la presencia del gen ureC, posee una solicitud de patente, la que se encuentra bajo el No 2016-01214, en INAPI (Instituto Nacional de Propiedad Industrial). En este trabajo, y por un protocolo similar al ya desarrollado, hemos determinado la prevalencia de las cepas cagA-positivo, en muestras fecales de pacientes sintomáticos digestivos de la Región de Coquimbo, infectados con $H$. pylori.

\section{Materiales y Métodos}

\section{Pacientes}

En este estudio participaron 160 pacientes sintomáticos digestivos, de los cuales 115 fueron mujeres $(71,9 \%)$ y 45 hombres $(28,1 \%)$, con una edad promedio de 55,6 años $( \pm 11,8)$, quienes fueron sometidos a una endoscopía digestiva alta de rutina. Las biopsias gástricas fueron obtenidas en forma protocolizada, tomando 2 muestras de antro distal (una en curvatura mayor y otra en curvatura menor), y 2 muestras de cuerpo (una de cara anterior y otra de cara posterior) durante la endoscopía. La determinación de $H$. pylori se realizó mediante tinción de Giemsa en la Unidad de Anatomía Patológica del Hospital San Pablo de Coquimbo, Chile.
El Comité de Bioética del Servicio Salud Coquimbo aprobó el protocolo y los pacientes firmaron voluntariamente el consentimiento informado.

\section{Extracción de DNA fecal y nested-qPCR}

Cada participante debió depositar alrededor de $5 \mathrm{~g}$ de heces en un frasco cerrado, que contenía 3 ml de RNA Later ${ }^{\circledR}$ (Ambion), el cual se almacenó a $-80{ }^{\circ} \mathrm{C}$ hasta su análisis.

Se utilizaron $200 \mathrm{mg}$ de cada muestra fecal para extraer el DNA, mediante el kit QIAamp ${ }^{\circledR}$ Fast DNA Stool Mini (QIAGEN), de acuerdo a las condiciones del fabricante. La integridad del DNA fue determinada mediante un gel de agarosa a $1 \%$ y su concentración determinada en un Nanodrop ${ }^{\mathrm{TM}}$ One (Thermo Scientific ${ }^{\mathrm{TM}}$ ). Luego, 120 ng de este DNA se utilizaron para amplificar los genes ureC o cagA, mediante nested-qPCR, proceso que comienza con una primera amplificación mediante PCR convencional, que se llevó a cabo en un Axygen ${ }^{\circledR}$ MaxyGene Thermal Cycler II. El DNA se incubó en presencia de $5 \mu \mathrm{l}$ Buffer $5 \mathrm{x} ; 1,5 \mu \mathrm{L} \mathrm{MgCl}_{2} 25 \mathrm{mM} ; 0,5 \mu \mathrm{L}$ dNTPs $10 \mathrm{mM}$; $1 \mu \mathrm{L}$ de cada partidor externo $(10 \mu \mathrm{M} c / \mathrm{u}), \mathrm{y} 0,2$ $\mu \mathrm{L}$ de Platinum Taq ${ }^{\circledR}$ DNA polimerasa $(5 \mathrm{U} / \mu \mathrm{L})$ (Invitrogen), en un volumen final de $25 \mu \mathrm{L}$. Las condiciones de amplificación incluyeron 30 ciclos a $95{ }^{\circ} \mathrm{C}$ por $45 \mathrm{~s}, 57^{\circ} \mathrm{C}$ por $45 \mathrm{~s}$ y $72{ }^{\circ} \mathrm{C}$ por $45 \mathrm{~s}$. Posteriormente, $2 \mu \mathrm{L}$ de una dilución 10x de este primer PCR, fueron usados para la siguiente ronda de amplificación, mediante qPCR, en un Eco Real Time PCR (Ilumina ${ }^{\circledR}$ ). La mezcla de qPCR contenía $5 \mu \mathrm{L}$ de SYBR Green $2 \mathrm{x}$ (KAPA SYBR ${ }^{\circledR}$ FAST qPCR) y $0,1 \mu \mathrm{l}$ de cada partidor interno $(10 \mu \mathrm{M} c / u)$, en un volumen final de $10 \mu \mathrm{L}$. Las condiciones de amplificación incluyeron 40 ciclos a $95^{\circ} \mathrm{C}$ por 10 s y $60{ }^{\circ} \mathrm{C}$ por 30 s. Como control se utilizó DNA extraído de la cepa 26695 de H. pylori.

La secuencia de los partidores utilizados en este trabajo se muestra en la Tabla 1.

\section{Análisis de los datos}

Los datos fueron analizados utilizando el Software Eco v4.1 PCR System y el programa XLSTAT Versión 2.06, para calcular sensibilidad, especificidad, y valor predictivo positivo y negativo de la detección de infección por H. pylori en las muestras fecales. Se utilizó la prueba de Histología-Giemsa como referencia de infección. Se calculó prevalencia de cepas cagA-positivo en 
Tabla 1. Información de los partidores utilizados

\section{Secuencia (5'- $\left.3^{\prime}\right)$ de los partidores}

Partidores externos ureC FExt: 5'-AGCTATAAAGTGGGCGAGAG-3' RExt: 5'-ATTGCACCCGTTAGGCTCAT-3'

Partidores Internos ureC FInt: $5^{\prime}$ - GCGTTGGCAGTGCTAAAAGG-3' Rint: 5'-AGCCGTATCTAACACGATCC-3'

Partidores externos cagA

Partidores Internos cagA
FExt: 5'-AAGCGCTAGCCGATCTCAAG-3' RExt: 5'-TGCCCTACATCACCCAAACC-3'

FInt: 5'-ACAGGAGAAGTAGCTAGCC-3' RInt: 5'-TGCCCTACATCACCCAAAC-3'

\section{Tamaño del amplicón}

224 bp

$127 \mathrm{bp}$

$400 \mathrm{pb}$

$113 \mathrm{pb}$

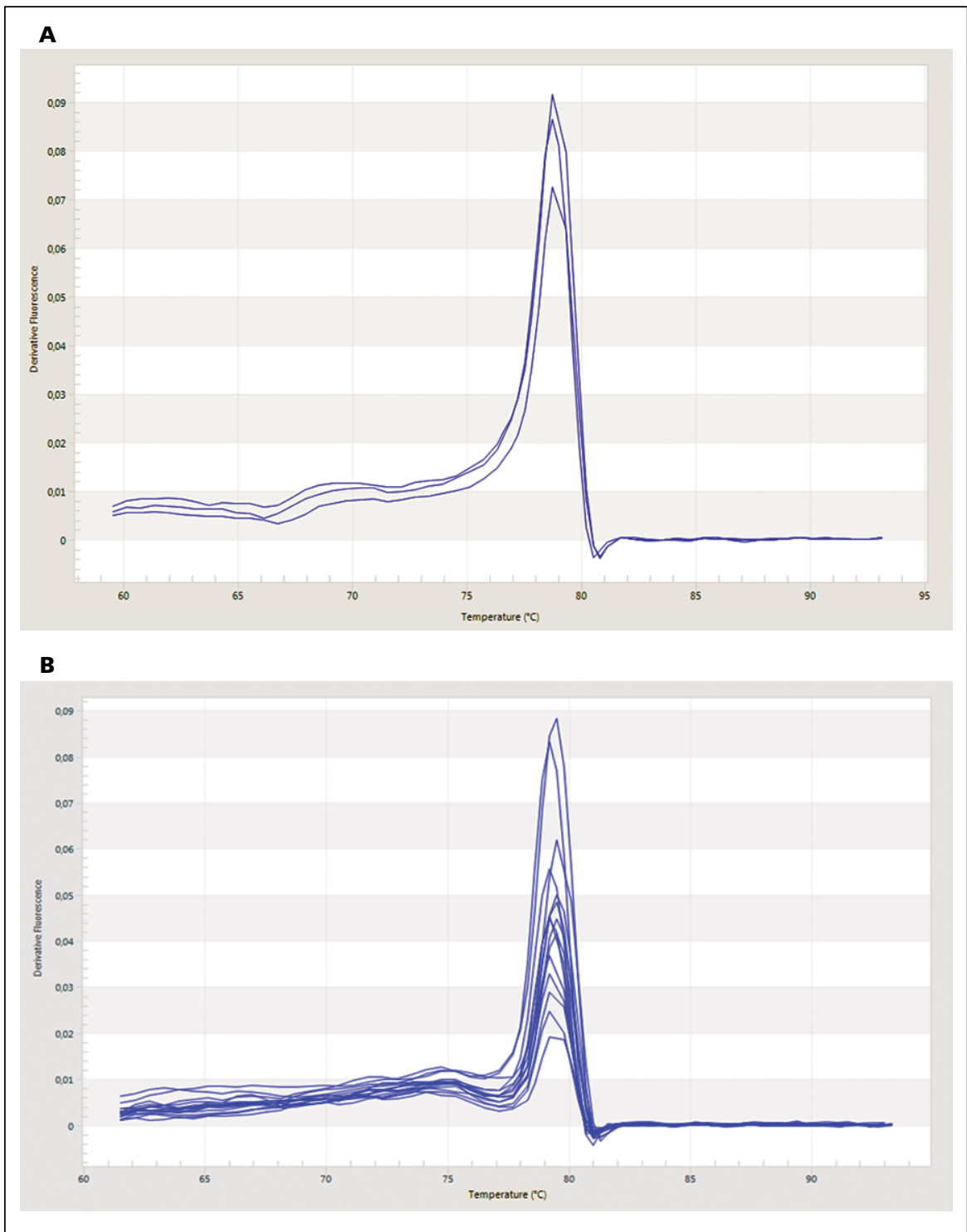

Figura 1. Curva de melt obtenida del software qPCR (Eco Real Time, Illumina) en que se muestra la amplificación del gen CagA desde la cepa control 26695 (A) o de un grupo de muestras fecales (B). Como se aprecia en la figura, el amplicón generado tiene una tm de $79^{\circ} \mathrm{C}$, el cual fue un indicador de positividad para este gen. 
Tabla 2. Distribución de la población, según características sociodemográficas y método diagnóstico

\begin{tabular}{|c|c|c|c|c|c|c|c|c|}
\hline \multirow[b]{2}{*}{ Mét. diagnóstico } & \multirow[t]{2}{*}{ n casos } & \multirow[t]{2}{*}{ (\%) } & \multicolumn{2}{|c|}{ Edad (años) } & \multicolumn{2}{|c|}{ Masculino } & \multicolumn{2}{|c|}{ Femenino } \\
\hline & & & Media / DS & Mín-Máx & $\mathbf{n}$ & $\%$ & $\mathbf{n}$ & $\%$ \\
\hline \multicolumn{9}{|l|}{ Histología } \\
\hline Positivo & 123 & 74,6 & $54,9 / 14,4$ & $17-86$ & 35 & 77,8 & 88 & 76,5 \\
\hline Negativo & 37 & 25,4 & & & 10 & 22,2 & 27 & 23,5 \\
\hline Total & 160 & 100 & & & 45 & 100,00 & 115 & 100,00 \\
\hline \multicolumn{9}{|l|}{ qPCR UreC } \\
\hline Positivo & 129 & 77,8 & $54,5 / 14,7$ & $17-86$ & 32 & 71,1 & 97 & 84,3 \\
\hline Negativo & 31 & 22,2 & & & 13 & 28,9 & 18 & 15,7 \\
\hline Total & 160 & 100 & & & 45 & 100,00 & 115 & 100,00 \\
\hline \multicolumn{9}{|l|}{ qPCR CagA } \\
\hline Positivo & 31 & 25,4 & $55,5 / 15,5$ & $22-86$ & 11 & 35,5 & 20 & 22,0 \\
\hline Negativo & 91 & 74,6 & & & 20 & 64,5 & 71 & 78,0 \\
\hline Total & 122 & 100 & & & 31 & 100 & 91 & 100 \\
\hline
\end{tabular}

Tabla 3. Parámetros estadísticos de la técnica nested-qPCR para la detección de $H$. pylori en muestras fecales

\begin{tabular}{|lc|}
\hline Parámetros estadísticos & $\%$ (IC) \\
\hline Sensibilidad & $96,7 \%(91,9 \%$ a $98,7 \%)$ \\
\hline Especificidad & $73,0 \%(57,0 \%$ a $84,6 \%)$ \\
\hline Valor predictivo (+) & $92,2 \%(86,3 \%$ a $95,7 \%)$ \\
\hline Valor predictivo (-) & $87,1 \%(71,1 \%$ a $94,9 \%)$ \\
\hline
\end{tabular}

la población evaluada sólo cuando la histología y el nested-qPCR ureC fueron positivos para esos pacientes. $\mathrm{p}<0,05$ fue considerado significante.

\section{Resultados}

Un total de 160 pacientes con sintomatología digestiva fueron evaluados para diagnosticar $H$. pylori en muestras fecales, y determinar la presencia de cepas virulentas cagA-positivo entre los infectados.

Mediante histología por Giemsa (método de referencia) se detectaron 123 pacientes infectados (76,9\%), 35 hombres (77,8\% del total de hombres) y 88 mujeres ( $76,5 \%$ del total de mujeres), mientras que se encontraron 129 pacientes infectados $(80,6 \%)$ utilizando nested-qPCR para ureC en muestras fecales; 32 hombres $(71,1 \%$ del total de hombres), y 97 mujeres (84,3\% del total de mu- jeres). La Sensibilidad de la técnica nested-qPCR para detectar la bacteria fue de 96,7\% (95\% CI: 91,9\%-98,7\%), mientras que la especificidad fue de 73,0\% (95\% CI: 57,0\%-84,6\%). A su vez, el VPP fue de $92,2 \%$ (95\% CI: $86,3 \%-95,7 \%)$ y el VPN fue de 87,1\% (95\% CI: 71,1\%-94,9\%). Según resultados obtenidos con el método de referencia, la infección por $H$. pylori tendría una prevalencia de $76,9 \%$ en la población evaluada.

Posteriormente, en 122 muestras positivas tanto para histología como para nested-qPCR ureC, se determinó la presencia de cepas cagA-positivo, encontrando 31 pacientes con esta cepa más virulenta, lo que corresponde a $25,4 \%$ de la población evaluada. No se consideraron para esta evaluación aquellas muestras en las que la histología fue positiva pero el nested-qPCR negativo para ureC, ni viceversa.

No se observó una relación significativa entre el género y la infección por $H$. pylori, ni tampoco entre género y prevalencias de cepas cagA-positivas.

Los resultados completos obtenidos en el diagnóstico de $H$. pylori con las distintas técnicas utilizadas, se encuentran en las Tablas 2 y 3.

\section{Discusión}

En este trabajo evaluamos la presencia de cepas cagA-positivo entre aquellos pacientes infectados con $H$. pylori en la región de Coquimbo, mediante nested-qPCR, a partir de DNA extraído 
desde muestras fecales ${ }^{15}$. Entre 160 pacientes con sintomatología digestiva, encontramos 123 en los cuales la Histología (Giemsa) detectó la bacteria, mientras que esta cifra aumentó a 129 pacientes cuando se utilizó nested-qPCR para el gen ureC. Estos resultados están de acuerdo a lo descrito en reportes previos, donde se establecen prevalencias de $76,8 \%$ y $73,0 \%$ respectivamente para $H$. pylori en Chile ${ }^{3,6}$.

En la población de la región de Coquimbo encontramos una prevalencia de $25,4 \%$ para cepas cagA-positivo entre los infectados por $H$. pylori, valor por sobre $15,2 \%$ encontrado recientemente por el grupo que dirige la Dra. Apolinaria García, en una población de la ciudad de Concepción ${ }^{16}$. Interesantemente, 16 años antes, en otra población de la misma ciudad de Concepción, una investigación encontró 38\% de cepas cagA-positivas entre los pacientes infectados con $H$. pylori ${ }^{17}$. Aparentemente, estos resultados se correlacionarían con la disminución observada en la tasa de mortalidad ajustada por cáncer gástrico en esta región, la que va de 28,37/100.000 hab. en el año 2000 , a 17,21/100.000 hab. en al año $2015^{18}$. En la región de Coquimbo, también se ha observado una disminución en la tasa de mortalidad ajustada por cáncer gástrico, la cual va de 21,59/100.000 hab en el año 2000, a 14,10/100.000 hab. en el año $2015^{18}$, lo cual podría, en parte, relacionarse con la baja tasa de cepas cagA-positivas observadas en este trabajo. No obstante, un mayor acceso de la población a las terapias de erradicación en los últimos años también podría haber favorecido esta disminución en la tasa de mortalidad registrada. $\mathrm{Al}$ respecto, de acuerdo al Maastricht V/Florence Consensus Report, se recomienda la estrategia de "diagnosticar y tratar" en poblaciones de alto riesgo de cáncer gástrico para la prevención de esta patología ${ }^{19}$.

Estos resultados son muy disímiles a los encontrados en la región del Maule, la que se caracteriza por una alta tasa de cáncer gástrico, en la cual $94,9 \%$ de los infectados con $H$. pylori, poseía la cepa cagA-positiva ${ }^{20}$. Esta prevalencia es muy similar a lo encontrado en Tailandia, donde 96,7\% de los infectados por el patógeno presentan la cepa cagA-positiva, no obstante, y de manera interesante, en este país la incidencia por cáncer gástrico no supera los 5/100.000 hab ${ }^{21}$. Otros estudios recientes muestran una prevalencia de $69,5 \%$, $77,3 \%$ y $87,5 \%$, para las cepas cagA-positivas en poblaciones de Malasia, India y China, respectivamente $^{22-24}$. Por su parte, en América Latina, se reportan similares resultados para este tipo de cepas, con prevalencias de 62,6\%, 71,0\% y $85,6 \%$, para México, Argentina y Brasil, respectivamen$\mathrm{te}^{25-27}$. Es interesante notar que reportes recientes no encuentran una asociación estadísticamente significativa para la prevalencia de cepas cagA-positivas y el cáncer gástrico ${ }^{24,25,27}$. Al respecto, es evidente que existen otros factores bacterianos asociados al riesgo de desarrollar cáncer gástrico, tales como la toxina vacuolizante VacA, entre otros, en particular la isoforma $\mathrm{s} 1 \mathrm{~m}^{24}$. De manera interesante, un trabajo reciente realizado en Chile demostró que cepas que portaban el gen vacAil tenían una fuerte correlación con lesiones gástricas severas, que podrían conducir al desarrollo de cáncer gástrico ${ }^{16}$. Otro factor que sin duda influye en la relación de las cepas cagA-positivas con el riesgo de cáncer gástrico, es la variación alélica en el motivo EPIYA en el carboxilo terminal de CagA. Cepas que contienen los alelos EPIYA-A, $-B$, y -D (provenientes de aislados de países de Asia del Este) muestran una mayor correlación con cáncer gástrico que cepas que contienen los alelos EPIYA-A, -B, y-C (provenientes de aislados de países occidentales $)^{28}$.

Por otro lado, y no menos importante, la edad de adquisición de la infección y los factores propios del huésped, son también condicionantes que podrían influir en el desarrollo de cáncer gástrico asociado a $H$. pylori $i^{29}$.

La técnica utilizada en este estudio no es invasiva, y el paciente solo debe enviar una muestra fecal, debidamente rotulada, al laboratorio, para su análisis, lo cual es una ventaja comparativa frente a las técnicas diagnósticas habituales. Al respecto, una revisión del año 2014 sugirió que la técnica de PCR podría ser superior a otros métodos diagnósticos para la detección de infección por $H$. pylori, debido a su alta sensibilidad y especificidad, especialmente con variantes que incluyen nested- $y$ semi-nested-PCR ${ }^{4}$.

Nuestros resultados son alentadores, puesto que esta técnica, no invasiva, económica y de alta sensibilidad, puede no sólo diagnosticar la infección por esta bacteria, sino que, como en este caso, determinar la presencia de cepas más virulentas en los pacientes. Actualmente, estamos incorporando al diagnóstico, otros genes asociados a virulencia, tales como vacAs $1 \mathrm{~m} 1$, y además, 
la determinación de cepas resistentes a claritromicina y metronidazol, a través del proyecto FONIS SA16I0230. Por otro lado, ya que la infección es adquirida durante la infancia, esta técnica podría ser convenientemente aplicada en estudios clínicos y epidemiológicos en población infantil.

En términos de costos, hemos calculado que la determinación de infección más la detección de cepas cagA-positivas en muestras fecales, tiene un valor aproximado de $\$ 10.000$ chilenos (USD 15,4).

Finalmente, estamos esperando la aprobación de nuestra solicitud de patente No 2016-01214 en INAPI para este protocolo diagnóstico.

Agradecimientos: Los autores agradecen al proyecto CORFO 12IDL2-16202.

\section{Referencias}

1. Burucoa C, Axon A. Epidemiology of Helicobacter pylori infection. Helicobacter 2017; 22: 1-5.

2. Ministerio de Salud. Tratamiento de erradicacion de Helicobacter pylori en el paciente con úlcera peptica. Serie guías Clínicas Chile 2013; 3-43. Available from: http://web.minsal.cl/portal/url/item/db8329eleffc9a22e040010165015626.pdf

3. Ferreccio C, Rollán A, Harris PR, Serrano C, Gederlini A, Margozzini P, et al. Gastric cancer is related to early Helicobacter pylori infection in a high-prevalence country. Cancer Epidemiol Biomarkers Prev 2007; 16: 662-7.

4. Patel SK, Pratap CB, Jain AK, Gulati AK, Nath G. Diagnosis of Helicobacter pylori: What should be the gold standard? World J Gastroenterol 2014; 20: 12847-59.

5. Hooi JKY, Lai WY, Ng WK, Suen MMY, Underwood FE, Tanyingoh D, et al. Global Prevalence of Helicobacter pylori Infection: Systematic Review and Meta-Analysis. Gastroenterology 2017; 153: 420-9.

6. Porras C, Nodora, J, Sexton R, Ferreccio C, Jiménez S, Domínguez RL, et al. Epidemiology of Helicobacter pylori infection in six Latin American countries (SWOG Trial S0701). Cancer Causes Control 2013; 24: 209-15.

7. Tsukamoto T, Nakagawa M, Kiriyama Y, Toyoda T, Cao X. Prevention of gastric cancer: Eradication of Helicobacter pylori and beyond. Int J Mol Sci 2017; 18: E1699.

8. Kim GH, Liang PS, Bang SJ, Hwang JH. Screening and surveillance for gastric cancer in the United States: Is it needed? Gastrointest Endosc 2016; 84: 18-28.

9. Jang B-G, Kim WH. Molecular pathology of gastric carcinoma. Pathobiology 2011; 78: 302-10.

10. Li H, Zhang B, Hu X, Dong Y, Fan Q, Guo F, et al.
Serum Helicobacter pylori FliD antibody and the risk of gastric cancer. Oncotarget 2016; 7: 22397-408.

11. Camilo V, Sugiyama T, Touati E. Pathogenesis of Helicobacter pylori infection. Helicobacter 2017; 22: 1-6.

12. Yong X, Tang B, Li B-S, Xie R, Hu C-J, Luo G, et al. Helicobacter pylori virulence factor CagA promotes tumorigenesis of gastric cancer via multiple signaling pathways. Cell Commun Signal. Cell Communication and Signaling 2015; 13: 30.

13. Hatakeyama M. Helicobacter pylori CagA and gastric cancer: A paradigm for hit-and-run carcinogenesis. Cell Host Microbe 2014; 15: 306-16.

14. Li Q, Liu J, Gong Y, Yuan Y. Association of CagA EPIYA-D or EPIYA-C phosphorylation sites with peptic ulcer and gastric cancer risks. Medicine (Baltimore) 2017; 96: e6620.

15. Taborda MI, Aquea G, Nilo Y, Salvatierra K, López N, López S, et al. Non-invasive diagnostic of Helicobacter pylori in stools by nested-qPCR. Polish J Microbiol 2018; 67: 11-18.

16. Paredes-Osses E, Sáez K, Sanhueza E, Hebel S, González C, Briceño C, et al. Association between cagA, vacAi, and dupA genes of Helicobacter pylori and gastroduodenal pathologies in Chilean patients. Folia Microbiol (Praha). Folia Microbiologica 2017; 62: 437-44.

17. Martínez A, González C, Kawaguchi F, Montoya R, Corvalán A, Madariaga J, et al. Helicobacter pylori: cagA status and vacA genotyping in Chile. Detection of a s2/ m1 strain. Rev Med Chile 2001; 129: 1147-53.

18. DEIS. Departamento de Estadísticas e Información de Salud. Ministerio de Salud 2017; http://www.deis.cl/ series-y-graficos-de-mortalidad/.

19. Malfertheiner P, Megraud F, O'Morain CA, Gisbert JP, Kuipers EJ, Axon AT, et al. Management of Helicobacter pylori infection-the Maastricht V/Florence Consensus Report. Gut 2017; 66: 6-30.

20. González I, Romero J, Rodríguez B, Llanos J, Morales E FH. High prevalence of virulence-associated genotypes in Helicobacter pylori clinical isolates in the Region del Maule, Chile. Scand J Infect Dis 2011; 43: 652-5.

21. Subsomwong P, Miftahussurur M, Vilaichone R, Ratanachu-ek T, Suzuki R, Akada J, et al. Helicobacter pylori virulence genes of minor ethnic groups in North Thailand Gut Pathog 2017; 9:56.

22. Osman HA, Hasan H, Suppian R, Hassan S, Andee DZ, Abdul Majid N, Zilfalil BA. Prevalence of Helicobacter pylori cagA, babA2, and dupA genotypes and correlation with clinical outcome in Malaysian patients with dyspepsia. Turk J Med Sci 2015; 45: 940-6.

23. Pandya HB, Agravat HH, Patel JS. Prevalence of Specific Helicobacter pylori cagA, vacA, iceA, ureC 
Genotypes and its Clinical Relevance in the Patients with Acid- Peptic Diseases. J Clin Diagn Res 2017; 11: DC23-DC26.

24. Pinto-Ribeiro I, Ferreira RM, Batalha S, Hlaing T, Wong SI, Carneiro F, et al. Helicobacter pylori vacA Genotypes in Chronic Gastritis and Gastric Carcinoma Patients from Macau, China. Toxins 2016; 8: 142.

25. Román-Román A, Martínez-Carrillo DN, Atrisco-Morales J, Azúcar-Heziquio JC, Cuevas-Caballero AS, Castañón-Sánchez CA, et al. Helicobacter pylori vacA s $1 \mathrm{~m} 1$ genotype but not cagA or babA2 increase the risk of ulcer and gastric cancer in patients from Southern Mexico. Gut Pathog 2017; 9: 18.

26. Medina ML, Medina MG, Merino LA. Correlation between virulence markers of Helicobacter pylori in the oral cavity and gastric biopsies. Arq. Gastroenterol 2017; 54: 217-221.

27. Vinagre ID, Queiroz AL, Silva Júnior MR, Vinagre RM, Martins LC. Helicobacter pylori infection in patients with different gastrointestinal diseases from northern Brazil. Arq Gastroenterol 2015; 52: 266-71.

28. Bridge DR, Blum FC, Jang S, Kim J, Cha J-H, Merrell DS. Creation and Initial Characterization of Isogenic Helicobacter pylori CagA EPIYA Variants Reveals Differential Activation of Host Cell Signaling Pathways. Sci Rep 2017; 7: 11057.

29. Dadashzadeh K, Peppelenbosch MP, Adamu AI. Helicobacter pylori Pathogenicity Factors Related to Gastric Cancer. Can J Gastroenterol Hepatol 2017; 2017: 7942489. 